\title{
II. Soziale Verflechtungen in England, Frankreich und den Niederlanden unter den Gesichtspunkten Hochadel, Beamten- schaft und Staat
}

\author{
Einleitung zur Diskussion
}

Antoni Maczak

\section{Patronage im Herzen des frühneuzeitlichen Europa}

Die Beiträge zu jenen Ländern, die als der nordwestliche Teil des europäischen Kernlandes definiert werden können, sind so unterschiedlich, wie vermutlich auch die jeweiligen Untersuchungsgegenstände. Denis Richet konzentriert sich auf einen Typus von Patronage-Beziehungen, der für die "gens de robe" charakteristisch war. Die Niederlande stellten sich in der fraglichen Zeit als ein besonders komplexes System von Fürstentümern dar, die vom 15. bis 17. Jahrhundert tiefgreifende politische Veränderungen erfuhren. Der Beitrag von Wim Blockmans konzentriert sich auf die kritische Periode nach der Katastrophe von Nancy, während Helmut Koenigsberger eine andere politische Krise und einen anderen methodischen Ansatz gewählt hat. Viktor Morgan schließlich präsentiert uns in äußerst prägnanter, auf die Beschreibung äußerer Gegebenheiten weitgehend verzichtender Form eine Fülle von Klientelsystemen und greift dabei die tiefverwurzelten Überzeugungen der eigenen Zunft in England heftig an.

Die meisten, vielleicht auch alle Formen und Funktionen von Patronage, die hier erwähnt werden, sind auch von anderen Ländern bekannt. Das interessante an Frankreich, den Niederlanden und England aber ist, daß dort eben jene verschiedenen Formen von Patronage in ein und demselben Land vorkommen. Das, was uns diese Beiträge zeigen, ist, daß gerade durch die Koexistenz verscbiedener Patronagearten das $\mathrm{Pa}$ tronage-Muster eines Landes bestimmt wird. Am Beispiel England zeigt sich dies besonders deutlich. Die Tudors bekämpften konsequent den Typus des Subklientelismus, der für die Mechanismen lokaler Einflußnahme so charakteristisch war. Dieser erinnert bisweilen an eine Art „bastard feudalism“, wie wir ihn aus einem Pachtvertrag 
in Wales (1585) kennen, demzufolge der Pächter ausschließlich die Tracht seines Herrn tragen und für ihn oder seinen Kandidaten stimmen mußte. In diesem besonderen Fall steht die Tracht als äußeres Zeichen von Loyalität für einen moderneren Typus des Klientelismus, der erst im „korrupten“ hannoverianischen Zeitalter zur Blüte gelangte. Lassen Sie mich nun - indem ich auf die Beiträge über Polen vorgreife - Ihre Aufmerksamkeit auf eine ziemlich auffallende Analogie zwischen den zeitgenössischen Wahlmechanismen in diesen, in anderer Hinsicht so unterschiedlichen Ländern lenken.

Ein umfassendes Wahlrecht, das in Polen für alle erwachsenen, als Adelige anerkannten Männer galt, trug stark zu einem Anwachsen von Patronage bei. Genauer gesagt, es stellte das Gleichgewicht her zwischen den Forderungen der möglichen Klienten nach Geld und materieller Unterstützung und dem Wunsch der Landbesitzer nach demonstrativer Unterstützung bei Wahlen. Es steht so gut wie außer Zweifel, daß gerade das liberale Wahlrecht die Bindungen Landadeliger untereinander, und zwar auch solcher, die in der Hierarchie weit voneinander entfernt waren, zumindest zeitweise, stärkte. In einigen Fällen bedeutete es für clevere und lokal einflußreiche Kleinadelige eine Art Druckmittel, um die Großen zu fetteren Geldgeschenken zu animieren (oder sogar zu erpressen). Dies muß nicht gleichermaßen charakteristisch für die Ära der ,alten Korruption“ in England sein, aber Hermann Wellenreuthers kürzlich erschienene, meisterhafte Studie über Landbesitzer und Wahlmechanismen hat mich von einer grundsätzlichen Analogie zwischen beiden Ländern hinsichtlich dieser besonderen Mechanismen überzeugt.

„Wahl-Patronage“ verdient mehr Aufmerksamkeit, weil sie - aber dies ist nur eine Hypothese - die Patrone zwang, ihre Klientelschaft über die täglichen ökonomischen Interessen hinaus zu erweitern. Morgan beweist mit seinem Beitrag Mut, wenn er die unterschiedlichen Arten von Patronage in ihrer wechselseitigen Beziehung diskutiert. Er zeigt auch, wie komplex dieses unser Thema ist, insbesondere wenn man versucht, die verschiedenartigen Klienteltypen mit ihren entsprechenden Rollen zu den jeweiligen Gesellschaften und Machtsystemen in Beziehung zu setzen.

Zu den bekanntesten Beispielen zählen dabei die Minister und Hofgünstlinge unter Königin Elisabeth und James I. Die Patronage-Mechanismen, die Walsingham, Burghley, Essex, Salisbury und Buckingham benutzten, waren einander ziemlich ähnlich. Dennoch gibt es sicher Historiker, die bestreiten würden, daß sie in ihrer Bedeutung für den Staat alle gleich waren. Einer der Hauptunterschiede bestand vermutlich in der Beziehung des einzelnen Patrons zum Herrscher, noch wichtiger aber war aller Wahrscheinlichkeit nach die jeweilige Auffassung von Politik und Staatsgeschäften. Es wäre wenig sinnvoll zu fragen, ob Burghley Elisabeth und Salisbury ihrem Nachfolger näherstanden als Buckingham dem letzteren. Das Problem ist, daß die Cecils viel mehr von Staatsraison verstanden (was immer damit gemeint sein mag) als der Villiers Clan. Ein ähnlicher, aber doch anders gearteter Fall begegnet bei Kardinal Richelieu, wo sich das Problem rivalisierender Patronage-Verbindungen bei Herrscher und Minister ebenfalls stellt.

Das Gesamtbild von Patronage-Strukturen in einzelnen Ländern wird nicht nur von den verschiedenen historischen Realitäten, sondern auch von den sehr unterschiedli- 
chen Einstellungen historischer Schulen zu diesem Thema bestimmt. In keinem anderen Land wurde Patronage so lange und genau studiert wie in Frankreich. In dieser Hinsicht sind wir alle Professor Roland Mousnier und seiner Schule zu großem Dank verpflichtet. Mousnier gehört in der Tat zu den wenigen Historikern, die sich im Rahmen ihrer Interpretation von Politik und Gesellschaft zu Beginn der Neuzeit ganz auf Patronage konzentrierten. Seine Schlußfolgerungen bezüglich Frankreich wurden in zahlreichen Fallstudien auf andere Länder des Westens ausgedehnt. Ich glaube nicht, daß Mousnier das Phänomen sozialer Verflechtung überbetont hat, vielmehr bin ich der Ansicht

1. daß man Standesgesellschaft und Patronage nicht als Beziehungsgeflecht betrachten sollte, das mit der Existenz sozialer Klassen an sich unvereinbar ist;

2. daß die Unterscheidung zwischen „maître-fidèle“ und „protecteur-créature“ bestenfalls ein Charakteristikum für Frankreich sein kann (wenn man zwischen beiden Begriffen überhaupt so scharf trennen will);

3. daß die Patron-Klient-Beziehung - in ihrer Wechselbeziehung von Professor Mousnier klar erkannt - unter bestimmten Bedingungen relativ flexibel war; ein Klient war sich bewußt, daß er mehreren Patronen "fidèle“ sein konnte, ebenso war ihm klar, daß er seine Loyalitäten wechseln konnte.

Ad.1 Die Frage von Patronage vs. Klassenunterschiede wird in unserer Diskussion erneut zur Sprache kommen. Es gibt darauf wahrscheinlich nicht nur eine Antwort, weil Patronage in manchen Fällen vorwiegend Nachbarschafts-Loyalitäten bezeichnet, bisweilen aber auch ein die ganze Nation umfassendes „broker“-System meint. Aber während eine Patronage, die man "Schutz“-Patronage nennen könnte, dazu tendiert, scharfe Klassenunterschiede auszugleichen und lokale Konflikte zu glätten, ähnelt eine gewaltsame Patronage, die vom stärkeren Partner erzwungen wird, nicht nur der Manifestation eines scharfen Klassenkonfliktes, sie ist vielmehr eine derartige Manifestation. Diese Art von Patronage liegt freilich außerhalb des französischen Forschungsfeldes über "fidélités“. Sie ist allerdings in italienischen Studien über Patronage relativ stark vertreten. Vor allem Giorgio Chittolini hat sich in dieser Hinsicht besondere Verdienste erworben.

Ad. 2 Nachfolgendes stellt eine Schlußfolgerung aus dem dar, was gerade über Gepflogenheiten der Patronagerhetorik gesagt wurde. Eine Vorbedingung für die Unterscheidung zwischen „fidèle" und „créature" ist ein fortgeschrittenes Stadium der Bürokratisierung, in dem „fidélité"-Beziehungen in hohem Maße auf Personen beschränkt sind, die nicht in das Netz öffentlicher und privater Ämterbeziehungen eingeschlossen sind. Sogar das französische Vokabular der Ära Richelieus scheint dieser Unterscheidung zu widersprechen. Nach Pontis, den Mousnier zitiert, wünschte Richelieu, daß seine Beamten nur ihm "fidèle“ wären; aber waren sie nicht seine „créatures“? Man wird, selbst wenn man die unterschiedlichen Formen von Höflichkeitsbezeugungen und Zeremoniell berücksichtigt, die jeweilige Zeit nicht dadurch besser verstehen, daß man das in den Quellen verwendete Vokabular zur Klassifizierung menschlicher Beziehungen benutzt. Auf diesem Wege wird man bestenfalls zu einer genaueren Erforschung der zeitgenössischen Phraseologie kommen. Man könnte fragen, ob diese Betonung, die auf die ausdrückliche Erklärung von Treue und Liebe gelegt wird, gerecht- 
fertigt ist. In welchem Maße spiegelt sie die wahre emotionale Verbundenheit des Klienten zum Patron wieder? Man darf natürlich eine echte emotionale Verbundenheit in einigen Fällen nicht ausschließen - ein Beispiel etwa ist Fabian Burggraf von Dohna. In seinen Memoiren beschreibt er höchst anschaulich nicht nur seine Zuneigung zu Heinrich IV. (und welcher fahrende Ritter dieser Zeit hätte dem „charme de son panache blanc" widerstehen können?), sondern auch seine Abneigung gegen seinen eigenen, früheren, kurzzeitigen Patron, Stefan Batory, den König von Polen ungarischer Abstammung (und gegen die polnische Nation im allgemeinen). Es scheint mir eine akzeptable Arbeitshypothese zu sein, daß viele (wenn nicht alle) Zeichen der Zuneigung gegenüber einer höhergestellten Person - dem Patron - nur Konvention waren. War es nicht, zumindest in einigen Fällen, ein zynischer Weg, eine erwünschte Patronagebeziehung herzustellen? Daß größte Vorsicht geboten ist, zeigen einschlägige Texte aus Polen-Litauen, wo die Rhetorik des Klientelismus gut entwickelt und sehr konventionell war.

Ad.3 Im Frankreich des frühen 17. Jahrhunderts war es irgendwie möglich, Klient zweier Herren zu sein, auch wenn diese in offenem Streit miteinander lebten. Da es den Kontrahenten in den seltensten Fällen darum zu tun war, ihre Sache baldmöglichst einer friedlichen Entscheidung zuzuführen, kann man nur schwer verstehen, wie ein Klient in derartigen Situationen mit seinen angeblich starken Gefühlen für seine Beschützer zurecht kam. Im Hoch- und Spätmittelalter waren die modernen Patronagebeziehungen denselben Veränderungen unterworfen wie die Feudalbeziehungen. Patronage verlor als Element hochgradig komplexer Beziehungsstrukturen auf einem bestimmten Grad der sozialen Hierarchie ihre Simplizität. Man kann annehmen, daß geteilte Loyalitäten kaum möglich waren, wenn Klienten in die Interessenssphäre ihrer Patrone integriert waren, so etwa, wenn sie von ihnen durch Pacht oder Besitz abhingen. Wo immer Klientelismus andererseits einer Dienst-Beziehung (im strengeren Sinne) ähnelte, konnte der Wechsel von Patronen zur Routine werden. Dies war in Kastilien der Fall, was Claude Marie Gerbet meisterhaft beschreibt, und auch an Magnatenhöfen in Polen vom 16. bis zum 18. Jahrhundert.

Für das Frankreich des 16. Jahrhunderts scheint charakteristisch gewesen zu sein, daß ein Landadeliger in seinem Schloß ein gutes Leben führen und dieses genießen konnte, so wie uns das Michel de Montaigne in höchsten Tönen preist. Der gleiche Landadelige tat aber vermutlich gut daran, sich um Protektion umzusehen, wenn Gefahr aufzog, vielleicht war er dann sein „vie retirée et particulière“ auch leid und wünschte, es aufzugeben. Klientelbeziehungen waren gang und gäbe; für die zweite Hälfte des 16. Jahrhunderts lassen sich Klientelcliquen auch deutlich abgrenzen. Was meines Wissens allerdings noch immer unklar bleibt, ist die Stabilität, die Dauerhaftigkeit derartiger Bande. Wie oft wurden Beziehungen dieser Art auf Grund veränderter konfessioneller Gegebenheiten neu geknüpft? Richets Primärinteresse richtet sich auf die Rolle der „robins“ im klientelen Bezugssystem und im besonderen auf deren Beziehungen zur "haute noblesse“. Einige Fragen bleiben jedoch offen. Wie üblich sind auch hier die Ursprünge des Phänomens besser bekannt als die Gründe seines Untergangs. $\mathrm{Zu}$ fragen bleibt: Was geschah mit der Patronage während der Fronde? Lloyd Mootes Buch über „A Revolt of the Judges“ erwähnt Patronage kaum; auch 
seine Rezensenten werfen diese Fragen nicht auf. Mme. Cubell war der Ansicht, daß „ce n'est pas un hasard (...) si la compagnie s'est alliée aux représentants les plus éminents de la noblesse française“ gegen die Politik Mazarins. „Leur alliance s'insère donc normalement dans le courant de révolte des privilégiés du royaume." Dies würde eine Gleichrangigkeit der Beziehung bedeuten, und zwar gleichrangig auf Grund der besonderen Stärke des „parlement“ als rebellierender Zusammenschluß in einer rebellierenden Stadt.

Der persönliche Herrschaftsstil Ludwigs XIV. zerstörte die meisten der traditionellen Strukturen des Klientelismus. Anders als seine Vorgänger und deren Kardinäle tolerierte der Sonnenkönig keine patrimonialen klientelen Beziehungen, die den Aufbau eines broker-Systems bedeutet hätten; ebensowenig benötigte er die Patronage seiner Minister und/oder Berater, um seinen Staat zu schützen. So wenigstens scheint es, weil die vor- und frühabsolutistische Ära in dieser Hinsicht viel genauer erforscht wurde. Aber schuf die absolutistische Bürokratie nicht ihre eigenen Formen von $\mathrm{Pa}$ tronage? Ludwig XIV. war der Patron. Wie groß aber war die Klientelpyramide, deren Spitze er darstellte?

Beide Beiträge, die den Niederlanden gewidmet sind, zeigen uns ein Land, das sich sehr von Frankreich und England unterscheidet. Blockmans konzentriert sich hauptsächlich auf das 15. Jahrhundert und besonders auf die mit dem unheilvollen Jahr 1477 in Zusammenhang stehenden Gegebenheiten. Zu dieser Zeit schien Patronage ein wichtiges Machtmittel in den Händen der burgundischen Regierung gewesen zu sein. Politische Unterstützung, die die Klienten boten, wurde als ein Teil des politischen Handelns verstanden. Die Käuflichkeit herzoglicher Ämter (weltlicher und kirchlicher) wurde von den Ständen (d.h. von den Städten) als dreiste Korruption betrachtet. Blockmans unterstreicht die Bedeutung des broker-Systems (des aktiven wie des passiven), das auf herzoglichem, kirchlichem oder sogar städtischem Patrimonium beruhte, er erwähnt aber auch, daß ,the great feudal lords could afford patronage with their own resources".

Dies könnte besonders wichtig in der späteren Periode gewesen sein, die von Professor Koenigsberger behandelt wird. Sein Thema ist in erster Linie der Kampf zwischen zwei politischen Patronen: Kardinal Granvelle und Wilhelm Prinz von Oranien. In dieser Zeit spielten - zumindest in den südlichen Provinzen der Niederlande - die Provinzgouverneure, die sich ihrer Familienverbindungen zum niederen Adel bedienten, die zentrale Rolle in den patrimonialen Beziehungssystemen. Die Krise in der Mitte des Jahrhunderts zwang die Adeligen, ihre Position zu verteidigen „by expanding their local power base and/or by attempting to capture control of their central government“. Beide Strategien ,hinged crucially on the control of patronage“. Der Hochadel schien mit seiner Fähigkeit, Freunde und Anhänger um sich scharen zu können, ziemlich zufrieden gewesen zu sein; dies war zumindest die Meinung Aerschots, und Granvelle glaubte, daß die Seigneurs immer noch großen Einfluß auf ihre sozial unter ihnen stehenden Nachbarn und selbst auf körperschaftlich organisierte Klienten, d.h. Stadtratsversammlungen, besaßen. Die allgemeine Frage, die hier gestellt werden sollte, ist die nach dem Alter und der Dauerhaftigkeit dieser Situation. Vielleicht noch allgemeiner: nach der Art und Weise, wie Patronagebeziehungen in 
Spannungszeiten und während politischer Krisen sich gestalteten. In einem kürzlich erschienenen Essay über Wilhelm von Oranien billigt Dr. Nierop der Patronage als einem Machtmittel der protestantischen Partei eher geringe Bedeutung zu. Möglicherweise bedarf Patronage, um existieren zu können, eines gewissen Grades von Stabilität oder zumindest der Aussicht auf Stabilität. Allerdings könnte man mit gleichem Recht auch sagen, daß potentielle Klienten gerade in Krisenzeiten und bei drohender Gefahr um Protektion nachsuchen. So ist es wohl besser, vorschnelle Generalisierungen zu vermeiden. Menschliche Reaktionen - in diesem Fall die Strategien der Klienten - hängen von zu vielen Variablen ab, um vorhersehbar zu sein. Dies mag zu einigem Zweifel an allzu weitreichenden Thesen über den Klientelismus berechtigen, Thesen vor allem, die von Sozialwissenschaftlern aufgestellt wurden, welche mit dem begrenzten Aussagewert ihrer Studien unzufrieden waren.

Doch bleibt noch immer die Frage, in welchem Ausmaß die Adelspatronage, wie sie Koenigsberger schildert, zurückprojeziert werden kann. Was geschah mit ihr während und nach der Befriedung der südlichen Provinzen der Niederlande durch die Spanier? Wurde sie durch einen anderen Patronagetypus mit anderen Patronen ersetzt?

Mein allgemeiner Eindruck von den beiden Beiträgen über die Niederlande ist, daß darin zwei verschiedene Länder behandelt werden, obwohl das eigentliche Unterscheidungsmerkmal die Chronologie und nicht die Geographie ist. Das hängt damit zusammen, daß beide Autoren sich auf sehr spezifische Situationen konzentrieren und daß sie bestimmte Typen der Beziehungen und bestimmte Aktionen in den Mittelpunkt stellen. Solche Fallstudien sind höchst aufschlußreich, und sie sollten eigentlich Hand in Hand gehen mit strukturell-typologischen Analysen, wie sie im Beitrag Victor Morgans geboten werden. So drängt sich eigentlich die Frage auf: Welche anderen Patronage-Beziehungen existierten noch in den Niederlanden und wie veränderten sie sich im Laufe der Zeit? Natürlich frage ich mich gleichzeitig, ob eine Antwort auf diese Frage unser wirkliches Ziel sein sollte.

Wir untersuchen die Patronage. Aber ich glaube nicht, daß wir es nur um der Patronage willen tun. Es könnte gefährlich sein, sich in sein bevorzugtes Forschungsthema zu verlieben. Die Patronage ist ein besonderes Thema auch deshalb, weil sie als eine Form der Macht stets in unterschiedlichen Verbindungen mit anderen Faktoren und Phänomenen erscheint. Ihre Typologie ist von Bedeutung nicht um ihrer selbst willen, sondern weil sie allgemeinere Veränderungen und Unterschiede gesellschaftlicher Beziehungen reflektieren könnte.

Eine Lektion, die wir aus der Entwicklung in den Niederlanden wohl lernen können, scheint zu sein (ich bin versucht, hier eine Hypothese zu wagen), daß, anders als in Großbritannien etwa, Patronage dort schon zu Beginn des 17. Jahrhunderts im Zuge der Modernisierung verschwand. Könnte es so gewesen sein, daß sie auf Grund der schnellen und tiefgreifenden Entwicklung der Geldwirtschaft veraltete? Oder daß starke Staatsstrukturen ihr ein Ende setzten? Oder darf man zwischen beiden Prozessen gar nicht trennen?

Künftiger Forschung möchte ich eine parallele Studie über Verflechtungen - oder Vernetzungen - in beiden Teilen der Niederlande des 17. Jahrhunderts vorschlagen. Möglicherweise wird uns ein Forscher, der sich mit niederländischer Wirtschaft be- 
schäftigt, eines Tages die Frage beantworten können, ob die ökonomische Expansion der vereinigten Provinzen zu dieser Zeit ohne Patronagebeziehungen ausgekommen ist; ob die wirtschaftlichen Verbindungen, wie sie zwischen den Amsterdamer Kaufleuten und ihren Partnern in Übersee bestanden, rein kommerziellen Charakter besaBen, und wie die Patrizier ihre Macht ausübten. 
